\title{
Efeito de um treinamento com realidade virtual não imersiva sobre a execução da tarefa motora real em idosos: relato de casos
}

\section{Effect of training with non-imersive virtual reality on the performance of the real motor task in the elderly: case reports}

\author{
Felipe Lima Rebêlo' ${ }^{1}$ (]) \\ Henrique Lima de Cerqueira Carvalho 2 (1) \\ Bárbara Pereira Fernandes $^{3}$ (1)
}

\author{
João Victor Pereira Barbosa 4 (1) \\ Alynne lasmin Batista Santos 5 (1) \\ Rodrigo da Silva Santos 6 (1)
}

\begin{abstract}
${ }^{1}$ Autor para correspondência. Universidade Estadual de Ciências da Saúde de Alagoas, Centro Universitário Cesmac (Maceió). Alagoas, Brasil. feliperebelo_fisio@yahoo.com.br

${ }^{2,3}$ Centro Universitário Cesmac (Maceió). Alagoas, Brasil. henrique.carvalho@cedu.ufal.br, bapereirafernandes@outlook.com 4-6Universidade Estadual de Ciências da Saúde de Alagoas (Maceió). Alagoas, Brasil. barbosa.jvictor@hotmail.com, iasminalynne@gmail.com, roddrigosilvva@gmail.com
\end{abstract}

\begin{abstract}
RESUMO | INTRODUÇÃO: No contexto de reabilitação em gerontologia surge como destaque a melhoria da capacidade de execução de tarefas motoras a fim de promover a autonomia, dessa maneira, é preciso investigar ferramentas para viabilizar esse objetivo clinico. OBJETIVO: Avaliar o efeito de um treino com realidade virtual não imersiva sobre a execução da tarefa motora real em idosos. MATERIAIS E MÉTODO: Trata-se de um relato de casos, com intervenção única, utilizando o Nintendo® Wii com sujeitos de idade superior a 60 anos submetidos à intervenção com treinamento de arremesso no jogo wii sports resort, e avaliados com analise cinemática angular de vídeo com o software Kinovea ${ }^{\circledR}$, antes e depois da intervenção, protocolo composto por uma bateria de dez arremessos, antes e após a intervenção, além da avaliação subjetiva da melhora pós-treino. RESULTADOS: Participaram da pesquisa 4 sujeitos, sendo 3 do sexo feminino, com média de idade 70,7 anos $\pm 7,13$ e escolaridade 8 anos $\pm 6,16$. Para as variáveis angulares verificou-se que, após o treino com Wii, houve acentuação na flexão de ombro e decréscimo no cotovelo para na postura inicial do arremesso; já na posição final do arremesso, ocorreu decréscimo na extensão de cotovelo e aumento da flexão de punho. Também foi verificado aumento do salto e diminuição no tempo de execução da tarefa. CONSIDERAÇÕES FINAIS: Diante disso é possível concluir que o Nintendo® Wii se mostra um recurso potencial para melhoria de gestos no âmbito real para idosos.
\end{abstract}

PALAVRAS-CHAVE: Aprendizado. Idoso. Biotecnologia.
ABSTRACT | INTRODUCTION: In the context of rehabilitation in gerontology, the improvement of the ability to perform motor tasks is highlighted to promote autonomy, so it is necessary to investigate tools to achieve this clinical objective. OBJECTIVE: To evaluate the effect of training with non-immersive virtual reality on the execution of the real motor task in the elderly. MATERIALS AND METHOD: This is a case report, with a single intervention using the Nintendo ${ }^{\circledR}$ Wii, with subjects over 60 years old who underwent intervention with throw training in the game Wii sports resort and evaluated with video angular kinematic analysis with the Kinovea ${ }^{\circledR}$ software before and after the intervention, a protocol consisting of a battery of ten shots before and after the intervention, in addition to the subjective assessment of posttraining improvement. RESULTS: Four subjects participated in the research, 3 females, with a mean age of 70.7 years \pm 7.13 and schooling 8 years \pm 6.16 . For the angular variables, it was found that, after training with the Wii, in the initial throwing posture, there was an accentuation in the shoulder flexion and a decrease in the elbow for the same movement in the final position. There was a decrease in the elbow extension and an increase in the wrist flexion. It was also verified an increase in the jump and a decrease in the task execution time. FINAL CONSIDERATIONS: Given this, it is possible to conclude that the Nintendo ${ }^{\circledR}$ Wii is a potential resource for improving gestures in real life for the elderly.

KEYWORDS: Learning. Aged. Biotechnology. 


\section{Introdução}

O aumento do número de idosos no Brasil e no mundo vem conduzindo uma mudança de paradigmas nos contextos políticos, científicos e sociais, traduzindo-se no desenvolvimento das políticas públicas que auxiliam ou contribuem para a melhoria da qualidade de vida dessa faixa etária. Associados a essas estratégias, surgiram também grandes avanços na tecnologia para promoção da saúde e reabilitação da população idosa. ${ }^{1-2}$ Esses recursos oferecem suporte acerca dos mais diversos aspectos que estão envolvidos no processo de envelhecimento. ${ }^{3}$

Nessa perspectiva, surge em 2005 um recurso de realidade virtual, inicialmente direcionado para o entretenimento, mas que logo foi incorporado aos programas de reabilitação, o exergame Nintendo ${ }^{\circledR}$ Wii (Foxconn, Taiwan), sendo utilizado na recuperação de diversos distúrbios e em diferentes faixas etárias, inclusive em pacientes idosos. ${ }^{4}$ Este recurso possui sensores capazes de captar os movimentos em três dimensões por meio de um giroscópio localizado no controle, que faz uso de tecnologia bluetooth para enviar as informações à uma estação (o console), sendo os movimentos realizados pelo jogador, contextualizado na realidade virtual não imersiva, nos diversos jogos existentes. 4

A utilização do Nintendo ${ }^{\circledR}$ Wii como recurso terapêutico vem sendo descrito na literatura como possibilidade na intervenção de diversas patologias como Parkinson, tratamento das sequelas motoras decorrentes de Acidente Vascular Encefálico e distúrbios de equilíbrio, sendo bem evidenciado como terapêutica efetiva e motivacional para o público idoso. . $-7^{-7}$

Em meio as discussões sobre a efetividade dessa nova tecnologia na área da saúde, surge o questionamento acerca do quanto o treinamento com essa realidade virtual é capaz de interferir em ganhos aplicáveis no ambiente real. Atualmente alguns autores defendem a ideia de que o treinamento de atividades motoras do cotidiano com o Nintendo ${ }^{\circledR}$ Wii não agregaria qualidade significativa ao movimento quando em seu contexto original.. Por outro lado, alguns estudos demonstram que o uso deste recurso é capaz de otimizar a capacidade funcional de idosos. ${ }^{9.10}$

A literatura ${ }^{11}$ defende que após a utilização de um treino com realidade virtual, alterações na biomecânica do movimento podem surgir já no primeiro dia de intervenção, devido às adaptações neurais que compõem o aprendizado motor, fenômeno não exclusivo da população jovem, onde alguns estudos ${ }^{12,13}$ evidenciam que apesar dos idosos sofrerem degeneração neuronal no processo de envelhecimento fisiológico (senescência), também é relatado a hipótese de aumento na arborização dendrítica possibilitando alterações plásticas como formação de novos engramas motores, diante de novas estimulações do ambiente externo.

Outro aspecto importante que também respaldaria a efetividade do treino virtual seria o tempo ótimo da tarefa (Timing). Alguns estudos apontam a redução do tempo de execução da atividade no ambiente real após o treino com realidade virtual, tanto em idosos quanto em jovens. 14

Diante disso, a relevância deste estudo se apresenta na medida em que busca refletir acerca dos aspectos biomecânicos e do timing da tarefa em relação ao treinamento virtual e sua consequência no ato motor em seu contexto real, contribuindo para a discussão acerca deste recurso. Assim, o objetivo do presente estudo foi avaliar o efeito de um treino com realidade virtual não imersiva sobre a execução da tarefa motora real em idosos.

\section{Métodos}

\section{Amostra}

Trata-se de um relato de casos realizado com idosos vinculados a um ambulatório de prevenção do risco de quedas de uma Unidade Docente Assistencial na cidade de Maceió, Alagoas. O estudo teve o protocolo aprovado pelo Comitê de Ética e Pesquisa do Centro Universitário CESMAC sob o número 1453/2012.

Foram incluídos indivíduos com idade igual ou superior a 60 anos, de ambos os sexos, sendo excluídos aqueles que apresentaram desordens clínicas que impossibilitassem a realização das atividades funcionais do protocolo utilizado na pesquisa, como alterações de locomoção, hipertensos não controlados e aqueles com instabilidade postural grave e déficit cognitivo, que foi avaliado pelo Mine Exame do Estado Mental. 
Em decorrência da especificidade do estudo e da população alvo, bem como as limitações físicas e dos recursos necessários para a coleta de dados, para esta pesquisa, optou-se por trabalhar com uma amostra não probabilística, por conveniência.

Os idosos foram recrutados no próprio espaço físi$\mathrm{co}$, onde acontecem as atividades do ambulatório de prevenção do risco de quedas.

\section{Procedimentos}

Inicialmente os pesquisadores explicaram aos idosos os procedimentos do estudo e posteriormente realizaram o convite de participação, frente a assinatura do Termo de Consentimento Livre e Esclarecido (TCLE).

O Mini Exame do Estado Mental, foi utilizado para avaliar a função cognitiva da amostra, trata-se de um instrumento amplamente utilizado em estudos nacionais e internacionais, e foi validado no Brasil por Bertolucci et al. ${ }^{15}$ É composto por um escore total de 30 pontos, e avalia orientação, memória imediata e de evocação, concentração, cálculo, linguagem e domínio espacial. Para os analfabetos, considera-se o ponto de corte de 18 pontos. Para aqueles com escolaridade entre um e quatro anos, adota-se o ponto de corte de 24 e acima de quatro anos, 26 pontos. Aqueles que apresentarem valores abaixo do ponto de corte apresentam provável déficit cognitivo.

Após a explicação dos procedimentos da pesquisa e assinatura do TCLE, os idosos foram encaminhados ao local de coleta de dados, uma sala reservada especificamente para este fim.

O estudo foi dividido em três momentos: Inicialmente, todos os participantes responderam a um questionário de dados socioeconômicos e demográficos: sexo, idade e escolaridade. Após a coleta inicial dos dados, os participantes foram encaminhados para um ginásio poliesportivo onde foi realizado a avaliação da tarefa funcional escolhida para este estudo (arremesso de uma bola de basquete). Na avaliação da tarefa o idoso era solicitado a arremessar duas baterias de 10 repetições sendo que só era considerado a segunda.

Os idosos foram posicionados a uma distância de 3,83 metros à frente do aro da cesta, que ficava numa altura de 3,74 metros do chão. Os movimentos realizados durante os arremessos foram filmados em três ângulos, através de três filmadoras Nikon coolpix P500 posicionadas frontalmente e nas laterais direita e esquerda. As três câmeras foram posicionadas de forma equidistantes a 3,43m do local dos arremessos. A escolha da metragem de distância foi definida devido ao favorecimento da análise dos movimentos dos sujeitos, pois só era possível gravar todo o corpo do sujeito com a distância anteriormente relatada.

Em um segundo momento, logo após a avaliação dos arremessos, os idosos foram submetidos à intervenção com o Nintendo ${ }^{\circledR}$ Wii, com duração de 20 minutos, através do jogo Wii Sports Resorts, modalidade esportiva basquete, que simula virtualmente o jogo de basquete através de arremessos reproduzidos pelos movimentos de membros superiores com o controle sem fio posicionado nas mãos do paciente.

Durante o treino com o Nintendo ${ }^{\circledR}$ Wii, todos os indivíduos foram colocados frente à televisão numa distância de 1 metro, com o monitor posicionado na altura dos olhos, sendo instruídos de forma padronizada durante a intervenção com foco na biomecânica de mobilidade de punho, saltos e extensão de membros superiores durante a tarefa. Ao final do treino virtual, os idosos repetiram a tarefa real (pós-teste), a qual foi mensurada seguindo a mesma metodologia da avaliação inicial (pré-teste).

Ao término do pós-teste, foi avaliada por meio de escala visual analógica (EVA) a percepção dos participantes a respeito da melhoria do desempenho na tarefa, onde os idosos eram solicitados a dar uma nota de zero a dez quanto à melhora em seu desempenho nos arremessos após o treino com o Nintendo ${ }^{\circledR}$ Wii, sendo zero uma nota que representaria nenhuma melhora, e dez, uma melhora excelente. Além disso, os mesmos eram questionados acerca de quais componentes eles perceberam melhora.

As análises cinemáticas dos movimentos foram realizadas através das filmagens com uso do Kinovea ${ }^{\circledR}$ (ARRUDA, Brasil), um programa de edição de vídeos projetado para analisar imagens e vídeos permitindo ainda a cronometragem do vídeo, aferição de movimentos angulares em cortes e marcação de pontos de referência. $\frac{16}{}$

Os pontos referenciais para as medições para cada articulação utilizada, do membro de dominância durante o arremesso, foram o terceiro metacarpo, processo estiloide da ulna, olecrano, acrômio e cristas ilíacas. 


\section{Análise Estatística}

Para apresentação dos dados sociodemográficos foi utilizado estatística descritiva no software Excel® 2003 (Microsoft, EUA) com média e desvio padrão.

Os dados de movimento angular obtidos por meio do software Kinovea® no pré e pós avaliação foram organizados em tabelas para apresentação com estatística descritiva sem aplicação de estatísticas inferencial devido ao tamanho da amostra.

\section{Resultados}

A amostra do estudo foi composta por 4 idosos, 3 do sexo feminino e 1 do sexo masculino. A média de idade encontrada foi de 70,7 anos ( $\mathrm{dp}= \pm 7,13$ ), sendo a mínima de 61, e a máxima 77 anos. A maioria dos sujeitos avaliados apresentou alta escolaridade, sendo a média de estudo de 8 anos $(\mathrm{dp}= \pm 6,16)$.

No fluxograma 1 abaixo é possível entender como se deu a captação dos participantes do estudo.

Fluxograma 1. Recrutamento dos participantes do estudo

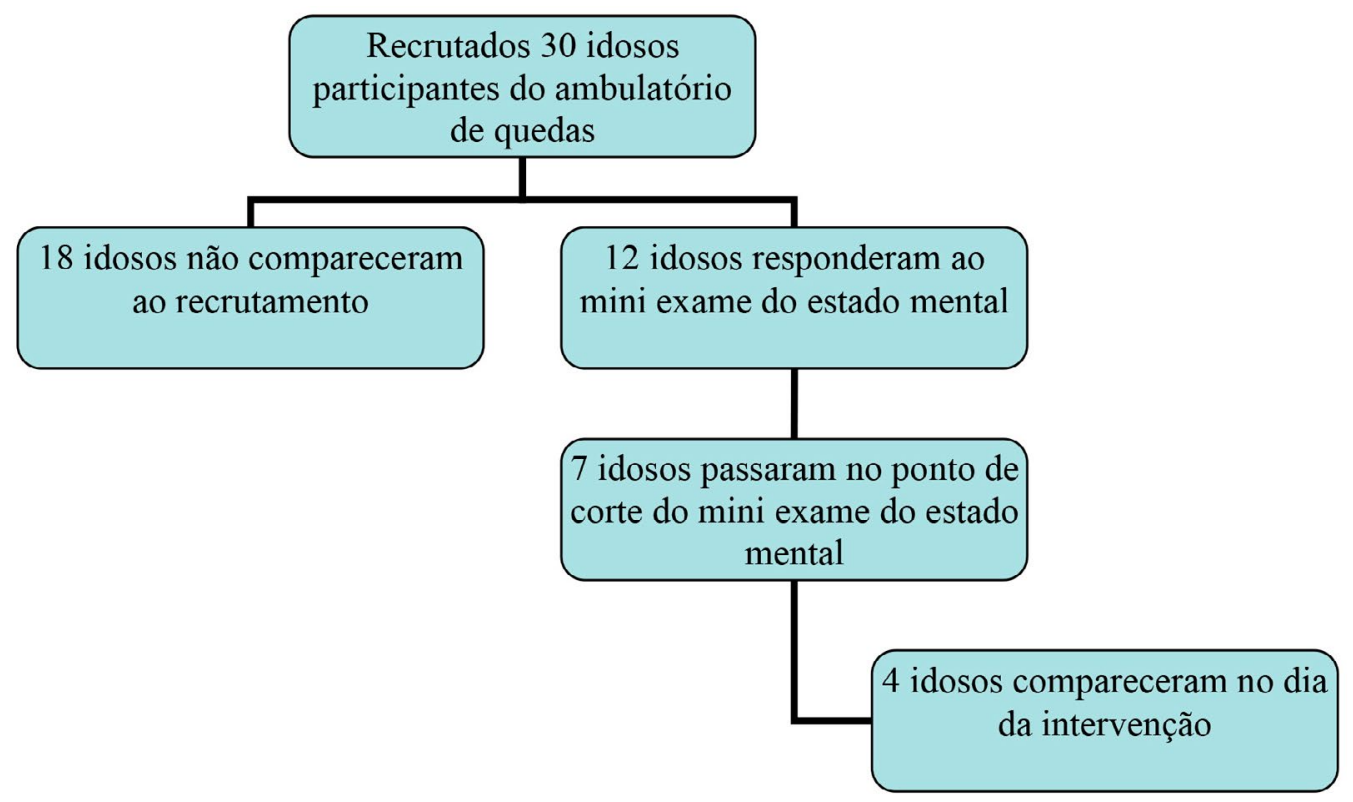

A apresentação dos dados referentes à avaliação biomecânica do movimento encontra-se distribuída em quatro tabelas. Na tabela 1, é possível observar o posicionamento angular do ombro, punho e cotovelo na posição inicial do arremesso realizado no basquetebol de costume com a bola acima da cabeça, comparando antes e após a intervenção com o recurso Wii. Foi observado, para os quatro idosos, um aumento angular nas articulações de ombro e cotovelo após a intervenção. No punho, verificou-se um aumento em três sujeitos. 
Tabela 1. Comparação da angulação na postura inicial do arremesso nas situações pré e pós intervenção nas articulações do punho, cotovelo e ombro

\begin{tabular}{cccccc}
\hline ARTICULAÇÃO & & Idoso 1 & Idoso 2 & Idoso 3 & Idoso 4 \\
\hline & Pré & 132,8 & 109,7 & 159,8 & 127,5 \\
PUNHO & Pós & 121,3 & 116,8 & 161,7 & 140,6 \\
COTOVELO & Pré & 58,4 & 50,2 & 57,4 & 66,8 \\
& Pós & 69,2 & 60,7 & 61,5 & 77,4 \\
& Pré & 45 & 43,2 & 82,3 & 78,9 \\
OMBRO & Pós & 64,3 & 46,8 & 88,8 & 107,2 \\
\hline
\end{tabular}

Fonte: Os autores (2021).

Na tabela 2, de forma semelhante, estão expressos a posição final do arremesso antes e após a intervenção para as mesmas articulações, onde verificou-se aumento angular no punho e diminuição no cotovelo em 3 sujeitos.

Tabela 2. Comparação da angulação na postura final do arremesso nas situações pré e pós intervenção nas articulações do punho, cotovelo e ombro

\begin{tabular}{cccccc}
\hline ARTICULAÇÃO & & Idoso 1 & Idoso 2 & Idoso 3 & Idoso 4 \\
\hline \multirow{2}{*}{ PUNHO } & Pré & 187,3 & 184,1 & 199,5 & 189,8 \\
& & & & 209,3 & 190 \\
COTOVELO & Pré & 179,6 & 187,5 & 149,8 & 142,8 \\
& & & 159,7 & 143,8 & 126,5 \\
OMBRO & Pós & 159,9 & 163,6 & 113,3 & 107,2 \\
& Pré & 108,1 & 102,9 & & 98,8 \\
\hline
\end{tabular}

Fonte: Os autores (2021).

Ainda em relação às variáveis angulares, estão expostos na tabela 3 os deltas angulares do movimento do arremesso (calculado pela subtração do valor da angulação na posição final da articulação pelo valor da angulação na posição inicial da mesma articulação), antes e após a intervenção. Para esta variável obteve-se valores menores para o cotovelo e ombro em três e quatro sujeitos respectivamente. 
Tabela 3. Comparação do delta angular nas situações pré e pós intervenção nas articulações do punho, cotovelo e ombro

\begin{tabular}{|c|c|c|c|c|c|}
\hline ARTICULAÇÃO & & Idoso 1 & Idoso 2 & Idoso 3 & Idoso 4 \\
\hline \multirow{3}{*}{ PUNHO } & Pré & 54,5 & 74,4 & 39,7 & 62,3 \\
\hline & Pós & 58,3 & 70,7 & 47,6 & 49,4 \\
\hline & Pré & 107,4 & 109,5 & 92,4 & 76 \\
\hline \multirow[t]{2}{*}{ COTOVELO } & Pós & 90,7 & 103,3 & 82,3 & 49,1 \\
\hline & Pré & 63,1 & 59,7 & 31 & 28,3 \\
\hline OMBRO & Pós & 48,4 & 63,9 & 15,6 & 8,4 \\
\hline
\end{tabular}

Fonte: Os autores (2021).

Na tabela 4, estão dispostos os resultados referentes ao tempo de execução e a altura do salto da tarefa motora antes e após o treinamento no Nintendo ${ }^{\circledR}$ Wii, onde verifica-se uma diminuição do tempo e aumento do salto em todos os sujeitos.

Tabela 4. Comparação do tempo de execução da tarefa motora e altura do salto nas situações pré e pós intervenção

\begin{tabular}{llllll}
\hline & & Idoso 1 & Idoso 2 & Idoso 3 & Idoso 4 \\
\hline \multirow{2}{*}{ Tempo (s) } & Pré & 24,3 & 20 & 21,1 & 24,9 \\
& Pós & 22,7 & 14,6 & 17,2 & 17,5 \\
\multirow{2}{*}{ Salto $(\mathrm{cm})$} & Pré & 15,32 & 5,97 & 5,97 & 9,91 \\
& Pós & 18,73 & 8,44 & 7,48 & 13,82 \\
\hline
\end{tabular}

Fonte: Os autores (2021).

Os dados subjetivos referentes à percepção de melhora dos participantes e do avaliador, descritos em duas categorias (sim ou não), bem como os resultados da escala analógica de percepção da melhora e os aspectos auto referenciados como melhoria pelos sujeitos do estudo encontram-se distribuídos na tabela 5. 
Tabela 5. Distribuição dos dados referentes as avaliações subjetivas

\begin{tabular}{lcc}
\hline Variável & Categoria & N \\
\hline $\begin{array}{c}\text { Percepção subjetiva de } \\
\text { melhora da qualidade }\end{array}$ & SIM & 4 \\
do arremesso pós \\
treino Wii (Avaliador)
\end{tabular}

Fonte: Os autores (2021).

\section{Discussão}

Os achados desse estudo remetem-se aos resultados de uma intervenção imediata e única com a Wiireabilitação em um grupo específico de idosos. Apesar de o grupo estudado ser pequeno, houve uma maior prevalência do sexo feminino que concorda com os demais estudos na área, que apontam as mulheres como o grupo que mais busca alternativas de prevenção em relação aos aspectos voltados à saúde e qualidade de vida. ${ }^{17}$ Mesmo com esse achado, é importante destacar que esse perfil passa atualmente por uma tendência de equiparação entre os sexos. 18

A alta escolaridade apresentada pode ser explicada pela natureza do grupo estudado, já que estes eram sujeitos que estavam inseridos em um grupo de atenção primaria à saúde, concordando com os achados de Lima-Costa ${ }^{19}$ que defendem a relação da alta escolaridade e melhores cuidados com a saúde, sendo representado no presente estudo pela participação em programa de prevenção.

Não foram encontrados estudos na literatura com metodologia semelhante, se tornando inviável comparações mais diretas, logo se torna relevante o apelo para novos estudos nesta perspectiva. Ainda assim, é possível realizar uma discussão em relação ao aperfeiçoamento da tarefa motora por meio do treino em realidade virtual com base na teoria de controle motor de Fitts e Posner. 20

Estes autores dividem o aprendizado motor em três fases: cognitiva, associativa e autônoma. Na primeira, ocorre o início da assimilação do gesto com presença de erros frequentes, porém sendo perceptíveis mudanças na qualidade do movimento desde a primeira intervenção. Na segunda, ocorre o refinamento da tarefa motora em aprendizagem, onde erros são menos frequentes. Já na terceira e última, ocorre a automação da habilidade motora com possibilidade de realização de outras tarefas simultaneamente, porém é valido salientar que esta pode demorar anos para se firmar. $\underline{21}$ 
Dessa forma, considerando o objeto desse estudo de avaliar a influência de um treino virtual imediato sobre a melhora na performance real, para a mesma atividade, fica claro a caracterização da fase cognitiva para o presente modelo experimental desta pesquisa. ${ }^{22}$ Logo, a provável ocorrência dessa fase durante a intervenção com a realidade virtual proporciona a transferência do controle motor adquirido virtualmente para o meio real.

A melhora nas diversas variáveis aqui apresentadas após o treino com o Nintendo ${ }^{\circledR}$ Wii podem ser atribuídas ao direcionamento dado no protocolo com intenso feedback para os movimentos do arremesso, como defende Wulf et al. ${ }^{21}$ Além disso, é provável que alterações plásticas a nível cortical já possam vir a ocorrer mediante a atenção seletiva do sujeito, que atua provocando atividade cortical intensa nos gânglios da base, como aborda Yarrow et al..22 Estudo realizado por Baumeister et al. 8 na Universidade de Paderborn, na Alemanha, com 10 golfistas profissionais, buscou comparar a atividade cortical na performance da tacada no ambiente real e virtual proporcionado pelo Nintendo ${ }^{\circledR}$ Wii, e para isso utilizou a leitura eletroencefalográfica simultânea à realização do gesto esportivo.

No entanto, os resultados confrontam com os achados desse estudo, já que os autores perceberam uma diferença na ativação cortical, concluindo que as atividades real e virtual não seriam semelhantes. Porém, é válido salientar que os sujeitos desta pesquisa foram jogadores experientes de golf, os quais já tinham engramas motores formados, muito provavelmente na terceira fase de aprendizado motor, o que implicaria que o contato com o Wii seria uma nova situação, logo, um novo processo de aprendizado motor. . $^{-}$

É válido salientar ainda que é referido na literatura ${ }^{22}$ que os idosos tendem a apresentar grandes dificuldades em passar pela terceira fase de aprendizado motor. Além disso, é sabido que essa faixa etária, diferentemente de atletas, por exemplo, não busca o auge no rendimento, mas sim um nível de melhoria funcional.

Na presente pesquisa, os achados evidenciaram meIhora significativa da angulação do ombro na posição inicial do arremesso, onde foi observado um aumento da flexão desta articulação após a intervenção. Em contra partida, os ângulos do cotovelo na posição inicial do arremesso após intervenção foram menores.
Isso pode ser explicado como uma consequência da maior flexão de ombro, pois caso esta modificação não fosse realizada, a bola seria posicionada atrás da cabeça, o que terminaria por dificultar a realização da atividade gestual ótima.

É possível atribuir a melhora no pós-treino à intervenção com o Wii, uma vez que o jogo escolhido utilizava elementos do lance livre do basquete com exigência de execução de movimento de punho e flexão de ombro, bem como deviam ser arremessados o máximo de bolas possíveis.

Desta forma, as alterações biomecânicas ocorridas com a intervenção do Nintendo ${ }^{\circledR}$ Wii podem ser entendidas como algo positivo, pois, como aponta Okazaki et al. $\underline{23}$, estas podem ocorrer para melhor controle e manutenção da precisão do ato motor e para aumento da velocidade do mesmo, o que é confirmado no presente estudo, onde houve redução do tempo de execução do movimento após a intervenção do a realidade virtual.

De maneira a acrescentar poder a esta discussão, também se evidencia o resultado da diminuição do delta angular das articulações do ombro e cotovelo, que acabam por corroborar para consequente diminuição do tempo de execução e menor angulação na posição final do cotovelo após a intervenção com a realidade virtual.

Também se evidenciou o aumento da flexão de punho no posicionamento final do arremesso após intervenção em três dos quatro sujeitos. Este achado corrobora o que foi elucidado anteriormente, reforçando a ideia da eficácia do treino virtual sobre a meIhora da percepção gestual no contexto real, pois vale ressaltar que durante o treino foi oferecido um feedback intenso ao movimento do punho, o que provavelmente contribuiu para estes resultados. Como explica Fitts e Posner ${ }^{20}$, é preciso uma retroalimentação intensa na fase cognitiva do aprendizado para que este seja mais efetivo

Outra variável que também sofreu melhora em todos os sujeitos foi a altura do salto, esta, por sua vez, possivelmente fortalece a ideia de aprendizado, já que não seria possível um ganho de força por mecanismos hipertróficos em uma única sessão, sobrando apenas as adaptações neurais como aumento no recrutamento de unidades motoras, melhoria no sinergismo e na reciprocidade muscular. $\underline{\underline{24}}$ 
As variáveis de percepção da atividade gestual foram positivas no sentido de melhora, tanto pelos participantes quanto pelos avaliadores, bem como a nota atribuída na escala visual analógica, fatores que também reforçam os referenciais teóricos discutidos anteriormente.

Chama-se a atenção para o autorrelato dos participantes da pesquisa inerentes aos componentes em que perceberam a melhora após o treino com o Nintendo ${ }^{\circledR}$ Wii. Os mesmos referenciaram melhora no movimento de punho e no salto no momento do arremesso, variáveis essas que sofreram grandes alterações entre o pré e o pós teste, o que remete a uma possível melhora na propriocepção do gesto esportivo, que é vista como parte integrante do aprendizado, já que seu aumento remete a um maior controle do corpo, oferecendo maior precisão na atividade motora, como defende Paiva et al..$\underline{5}$

É possível encontrar na literatura cientifica outros relatos da transferência do aprendizado no âmbito na realidade virtual para o contexto real; estes são os estudos do uso do recurso para melhoria de técnicas médicas em procedimentos cirúrgico, como em Paiva et al. ${ }^{25}$, sendo o último um ensaio randomizado em que foi verificado a possibilidade da transferência da habilidade do contexto virtual para o real. No entanto, é valido salientar que este modelo de treinamento é baseado em uma forma imersiva do meio diferenciando-se do Wii que é um recurso de realidade virtual do tipo não imersiva.

Apesar destas contribuições, é preciso a constante expansão de estudos com perspectivas semelhantes, pois como foi evidenciado, não foi possível realizar comparações diretas devido a essa escassez. O reduzido número de participantes terminou por não proporcionar a formação de um grupo controle e mesmo um maior número de participantes no grupo intervenção, diminuindo o grau de evidência científica desta contribuição. No entanto, essa pesquisa foi de grande valia para o pontapé inicial desta perspectiva para o público idoso.

\section{Considerações finais}

Com base nos achados da presente pesquisa, é possível sugerir que o uso do Nintendo ${ }^{\circledR}$ Wii como recurso terapêutico para melhoria do gesto motor gera diminuição no tempo de execução do mesmo, o que é possível pelas alterações biomecânicas verificadas, com foco nas articulações do ombro e punho, além da melhoria do salto.

\section{Contribuições dos autores}

Rebêlo FL, Carvalho HCL e Fernandes BP participaram da concepção, delineamento, busca e análise estatística dos dados da pesquisa, interpretação dos resultados, redação do artigo científico. Barbosa JVP, Santos AIB e Santos RS participaram da coleta e interpretação dos dados e do artigo científico.

\section{Conflitos de interesses}

Nenhum conflito financeiro, legal ou político envolvendo terceiros (governo, empresas e fundações privadas, etc.) foi declarado para nenhum aspecto do trabalho submetido (incluindo, mas não se limitando a subvenções e financiamentos, participação em conselho consultivo, desenho de estudo, preparação de manuscrito, análise estatística, etc.).

\section{Referências}

1. Oliveira AS. Transição Demográfica, Transição Epidemiológica $E$ Envelhecimento Populacional No Brasil. Hygeia. 2019;15(32):6979. http://dx.doi.org/10.14393/Hygeia153248614

2. Costa ARGP, Bessa MEP, Oliveira FCMB. Tecnologias utilizadas na assistência domiciliar ao idoso. R Saúd Digi Tec Edu [Internet]. 2019;4(2):131-43. Disponível em: http://www.repositorio.ufc.br/ handle/riufc/49174

3. Woll A, Bratteteig T. A trajectory for technology-supported elderly care work. Comput Support Coop Work. 2019;28(1-2):12768. https://doi.org/10.1007/s10606-018-9340-2

\section{Shih $\mathrm{CH}$, Chang ML, Mohua Z. A three-dimensional object orientation detector assisting people with developmental disabilities to control their environmental stimulation through simple occupational activities with a Nintendo Wii Remote Controller. Res Dev Disabil. 2012;33(2):484-9. https://doi. org/10.1016/j.ridd.2011.10.012}

5. Garcia-Agundez A, Folkerts A-K, Konrad R, Caserman P, Tregel T, Goosses $M$, et al. Recent advances in rehabilitation for Parkinson's Disease with Exergames: A Systematic Review. J Neuroeng Rehabil. 2019;16(1):17. https://doi.org/10.1186/s12984-019-0492-1

6. Bueno LS, Araújo RA, Oliveira MAF. Efeitos da prática do Yoga no medo de cair e mobilidade de idosos caidores. Re Cient Sena Aires. 2019;8(4):382-93. https://doi.org/10.36239/revisa.v8.n4. p382a393 
7. Mézière A, Denis A, Berchel N, Moreau C, Perrot A. Psychological impact of Wii-empowerment in hospitalized elderly patients who fall: pilot study. Soins Gerontol. 2020;25(142):34-8. https://doi.org/10.1016/j.sger.2020.01.009

8. Baumeister J, Reinecke K, Cordes M, Lerch C, Weiß M. Brain activity in goal-directed movements in a real compared to a virtual environment using the Nintendo Wii. Neurosci Lett. 2010;481(1):47-50. http://dx.doi.org/10.1016/j.neulet.2010.06.051

9. Sposito LAC, Portela ER, Bueno EFP, Carvalho WRG, Silva FF, Souza RA. Experiência de treinamento com Nintendo Wii sobre a funcionalidade, equilíbrio e qualidade de vida de idosas. Motriz rev educ fís. 2013;19(2):532-40. http://dx.doi.org/10.1590/S198065742013000200031

10. Wibelinger LM, Batista JS, Vidmar MF, Kayser B, Pasqualotti A, Schneider RH. Efeitos da fisioterapia convencional e da wiiterapia na dor e capacidade funcional de mulheres idosas com osteoartrite de joelho. Rev Dor. 2013;14(3):196-9. http://dx.doi. org/10.1590/S1806-00132013000300009

11. Doyon J, Penhune V, Ungerleider LG. Distinct contribution of the cortico-striatal and cortico-cerebellar systems to motor skill learning. Neuropsychologia. 2003;41(3):252-62. https://doi. org/10.1016/S0028-3932(02)00158-6

12. Wisniewski HM, Merz GS. Neuropathology of aging brain [Internet]. In: Gaitz CM, Samorajski T, editores. Aging 2000: Our Health Care Destiny. Nova lorque: Springer-Verlag; 1985. p. 23143. Disponível em: https://link.springer.com/book/10.1007/978-14612-5058-6

13. Pellicciari MC, Miniussi C, Rossini PM, De Gennaro L. Increased cortical plasticity in the elderly: changes in the somatosensory cortex after paired associative stimulation. Neuroscience. 2009;163(1):266-76. http://dx.doi.org/10.1016/j. neuroscience.2009.06.013

14. Santos S, Tani G. Tempo de reação e aprendizagem de uma tarefa de "timing" antecipatório em idosos. Rev paul educ fis. 1995;9(1):51-62. https://doi.org/10.11606/issn.2594-5904. rpef.1995.139418

15. Brucki SMD, Nitrin R, Caramelli P, Bertolucci PHF, Okamoto $\mathrm{IH}$. Sugestões para o uso do mini-exame do estado mental no Brasil. Arq Neuropsiquiatr. 2003;61(3B):777-81. http://dx.doi. org/10.1590/S0004-282X2003000500014
16. Cirne V. Análise de Posturas e de Movimento com Recurso a um Método de Análise de Imagem [dissertação] [Internet]. Porto: Universidade do Porto; 2013. Disponível em: https://repositorioaberto.up.pt/bitstream/10216/69657/2/27263.pdf

17. Alves RF, Silva RP, Ernesto MV, Lima AGB, Souza FM. Gênero e saúde: o cuidar do homem em debate. Psicol teor prat [Internet]. 2011;13(3):152-66. Disponível em: http://www.redalyc.org/ articulo.oa?id=193821358012

18. Borges LM, Seidl EMF. Percepções e comportamentos de cuidados com a saúde entre homens idosos. Psicol Cienc Prof. 2012;32(1):66-81. http://dx.doi.org/10.1590/S1414$\underline{98932012000100006}$

19. Lima-Costa MF. A escolaridade afeta, igualmente, comportamentos prejudiciais à saúde de idosos e adultos mais jovens? - Inquérito de Saúde da Região Metropolitana de Belo Horizonte, Minas Gerais, Brasil. Epidemiol Serv Saúde. 2004;13(4):201-8. http://dx.doi.org/10.5123/S167949742004000400002

20. Fltts PM, Posner MI. Human performance. Belmont, CA: Brooks/Cole; 1967. Disponível em: http://www.garfield.library. upenn.edu/classics1985/A1985ADR7200001.pdf

21. Wulf G, Chiviacowsky S, Schiller E, Ávila LTG. Frequent external-focus feedback enhances motor learning. Front Psychol. 2010;1:190. https://doi.org/10.3389/fpsyg.2010.00190

22. Yarrow K, Brown P, Krakauer JW. Inside the brain of an elite athlete: The neural processes that support high achievement in sports. Nat Rev Neurosci. 2009;10(8):585-96. http://dx.doi. org/10.1038/nrn2672

23. Okazaki VHA, Okazaki FHA, Rodacki ALF, Lima AC. Variabilidade inter-individual na estrutura temporal do arremesso no basquetebol. Motriz [Internet]. 2009;15(4):831-41. Disponível em: http://www.academia.edu/download/42659925/Variabilidade_ inter-individual na estrut20160213-27515-1 viwret.pdf

24. Heuninckx S, Wenderoth N, Debaere F, Peeters R, Swinnen SP. Neural basis of aging: The penetration of cognition into action control. J Neurosci. 2005;25(29):6787-96. https://doi.org/10.1523/ JNEUROSCI.1263-05.2005

25. Paiva PVF, Machado LS, Santos SR, Romero ROG. Potencialidades da Educação em Saúde Baseada em Realidade Virtual. Revista Eletrônica de Comunicação, Informação e Inovação em Saúde [Internet]. 2013;7(3):1-16. https://www.reciis. icict.fiocruz.br/index.php/reciis/article/view/540 